\title{
The rp-Process in Core-collapse Supernovae
}

\section{Shinya Wanajo*}

Department of Astronomy, School of Science, University of Tokyo, Bunkyo-ku, Tokyo, 113-8654 E-mail: wanajodastron.s.u-tokyo.ac.jp

Recent hydrodynamic simulations of core-collapse supernovae with accurate neutrino transport suggest that the bulk of the neutrino-heated ejecta is proton rich, in which the production of some interesting proton-rich nuclei is expected. However, there are a number of waiting point nuclei with the $\beta^{+}$-lives of a few minutes, which prevent the production of heavy proton-rich nuclei beyond iron in explosive events such as core-collapse supernovae. In this study, it is shown that the rapid proton-capture $(r p)$ process takes place by bypassing these waiting points via neutroncapture reactions even in the proton-rich environment, if there is an intense neutrino flux as expected during the early phase of the neutrino-driven winds of core-collapse supernovae. The nucleosynthesis calculations imply that the neutrino-driven winds can be potentially the origin of light $p$-nuclei including ${ }^{92,94} \mathrm{Mo}$ and ${ }^{96,98} \mathrm{Ru}$, which cannot be explained by other astrophysical sites.

International Symposium on Nuclear Astrophysics - Nuclei in the Cosmos - IX

June 25-30 2006

CERN, Geneva, Switzerland

${ }^{*}$ Speaker. 


\section{INTRODUCTION}

The rapid proton-capture ( $r p$ ) process has been expected to take place in proton-rich compositions with sufficiently high temperature [1] such as in X-ray bursts [2], whose nucleosynthetic products might contribute to the Galactic chemical evolution of some proton-rich isotopes including $p$-nuclei. Recent hydrodynamic simulations of core-collapse supernovae with accurate neutrino transport show that the bulk of the neutrino-heated ejecta during the early phase is proton rich [3], in which the production of some proton-rich nuclei is expected. In such environments, the waiting point nuclei (e.g., ${ }^{64} \mathrm{Ge}$ ) are bypassed via neutron capture reactions even in proton-rich matter, as first pointed out by [5] ( $v p$-process). As a result, the $r p$-process-like nucleosynthesis takes place that leads to the production of proton-rich nuclei beyond $A \sim 100$ [4, 6]. A more detailed discussion of the current results is presented in [6].

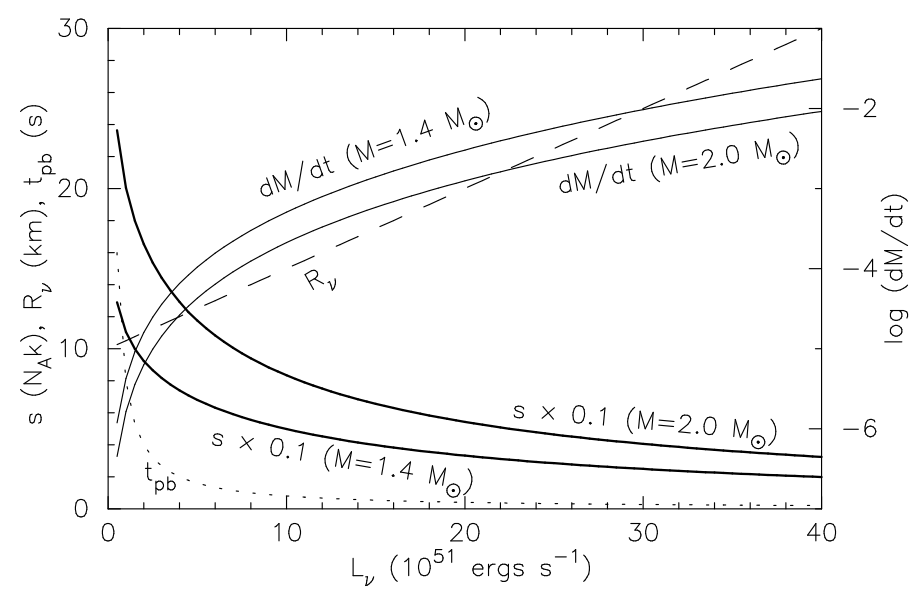

Figure 1: Model parameters $\left(R_{v}, t_{\mathrm{pb}}, \dot{M}\right)$ are shown as functions of $L_{v}$. Also denoted are the obtained entropies for $1.4 M_{\odot}$ and $2.0 M_{\odot}$ cases.

\section{NEUTRINO-DRIVEN WINDS}

Assuming the spherical symmetry of the neutrino-driven winds from a nascent neutron star, the equations of baryon, momentum, and mass-energy conservation with the Schwarzschild metric can be solved numerically [7, 8, 9]. Once the neutron star mass $(M)$, the neutrino sphere radius $\left(R_{v}\right)$, and the neutrino luminosity $\left(L_{v}\right.$, of one flavor, where each flavor is assumed to have the same luminosity) are specified along with the mass ejection rate $(\dot{M})$ as the boundary condition, the wind solution can be obtained. In this study, the neutron star mass is taken to be $1.4 M_{\odot}$ and $2.0 M_{\odot}$.

The time evolutions of $L_{v}$ and $R_{v}$ are assumed to be $L_{v}\left(t_{\mathrm{pb}}\right)=L_{v 0}\left(t_{\mathrm{pb}} / t_{0}\right)^{-1}$ and $R_{v}\left(t_{\mathrm{pb}}\right)=$ $\left(R_{v 0}-R_{v f}\right)\left(t_{\mathrm{pb}} / t_{0}\right)^{-1}+R_{v f}$, where $t_{\mathrm{pb}}$ is the post-bounce time, $t_{0}=0.2 \mathrm{~s}, L_{v 0}=4 \times 10^{52} \mathrm{ergs} \mathrm{s}^{-1}$, $R_{v 0}=30 \mathrm{~km}$, and $R_{v f}=10 \mathrm{~km}$, which mimic the hydrodynamic results of the neutrino-driven winds in [10]. The wind trajectories are calculated for 54 constant $L_{v}$ between 0.5 and $40 \mathrm{ergs} \mathrm{s}^{-1}$. Figure 1 shows the obtained entropies in winds for $1.4 M_{\odot}$ and $2.0 M_{\odot}$ cases as functions of $L_{V}$, along with the morel parameters $\dot{M}, R_{v}$, and $t_{\mathrm{pb}}$. 


\section{NUCLEOSYNTHESIS}

The nucleosynthetic yields for each wind trajectory are obtained by application of an extensive nuclear reaction network that consists of $\sim 6300$ species between the proton and neutron drip lines along with all relevant nuclear reaction and weak rates [11]. The neutrino captures on free nucleons are also included. Each calculation is initiated $(t=0 \mathrm{~s})$ when the temperature decreases to $T_{9}=9$ (where $T_{9} \equiv T / 10^{9} \mathrm{~K}$ ).
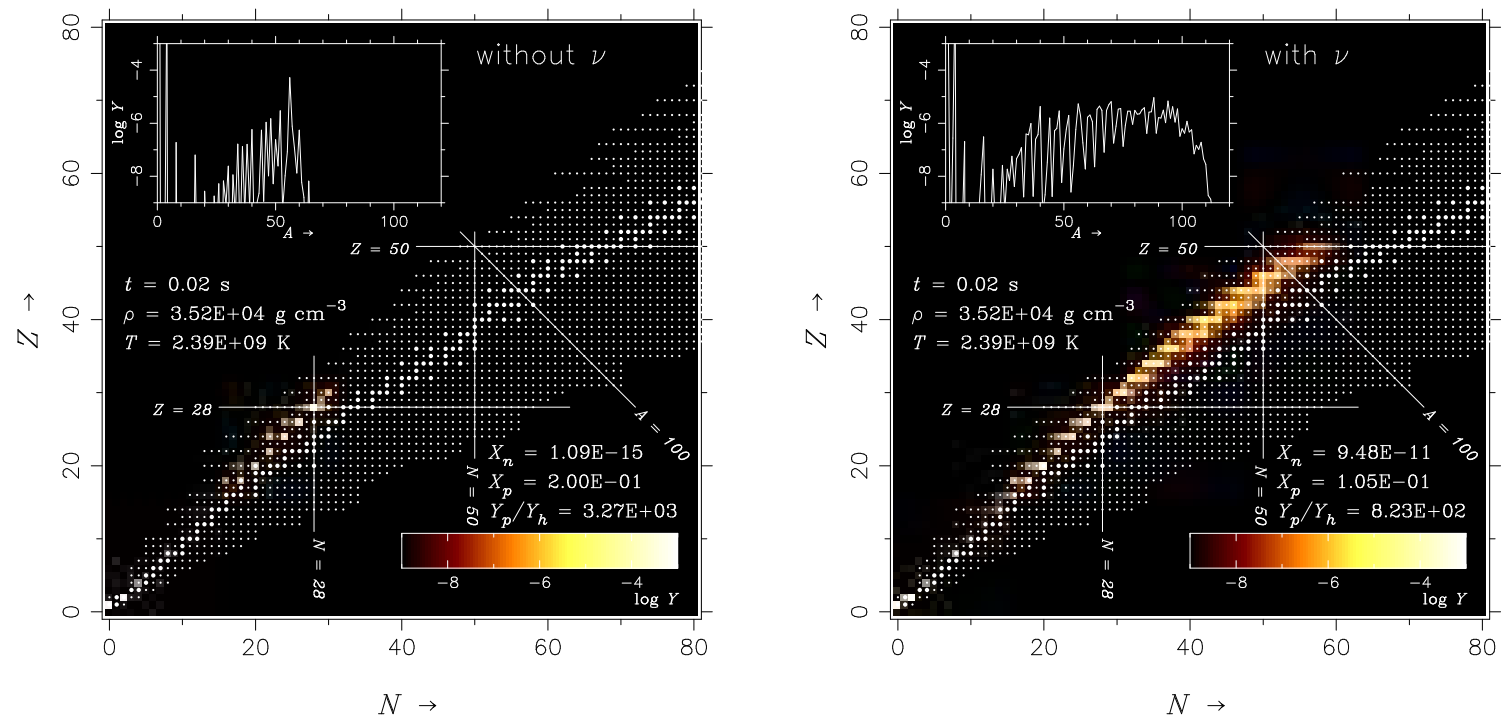

Figure 2: Snapshot of the nucleosynthesis at $t=0.02 \mathrm{~s}$ for the wind trajectory of $M=2.0 M_{\odot}, L_{v}=4.0 \times$ $10^{51} \mathrm{ergs} \mathrm{s}^{-1}, t_{\mathrm{pb}}=2.0 \mathrm{~s}$, and $Y_{e i}=0.600$, where the neutrino reactions are turned off (left panel) and on (right panel). The abundances are shown by the image in the nuclide chart. The abundance curve as a function of mass number is shown in the upper left for each panel.

Figure 2 shows the snapshot of nucleosynthesis at $t=0.02 \mathrm{~s}$ for the wind trajectory of $M=$ $2.0 M_{\odot}, L_{v}=4.0 \times 10^{51} \mathrm{ergs} \mathrm{s}^{-1}, t_{\mathrm{pb}}=2.0 \mathrm{~s}$, and $Y_{e i}=0.600$, where the neutrino reactions are turned off (left panel) and on (right panel). $Y_{e i}$ is the initial electron fraction (number of proton per baryon) at $T_{9}=9(t=0 \mathrm{~s})$. The entropy obtained for this trajectory is $129 N_{A} k$. Without neutrino reactions, the abundances have a sharp peak at ${ }^{56} \mathrm{Ni}$ and the nuclear flow stops at ${ }^{64} \mathrm{Ge}$ owing to its long half life (1.062 $\mathrm{min})$ as well as the small proton-separation energies around these species. In contrast, the inclusion of neutrino reactions leads to the nuclear flow reaching the $Z=50$ proton-magic number, resulting in the production of proton-rich isotopes beyond $A=100$. This neutrino-induced rp-process is a consequence of the presence of neutrons formed by the capture of anti-electron neutrinos on free protons. As a result, the waiting point nuclei are bypassed via neutron captures $(n, p)$ and $(n, \gamma)$, and the proton capture proceeds.

The time evolution of $Y_{e i}$ that determines the initial composition for each wind trajectory is assumed as in the top panel of Figure 3, so as to mimic the hydrodynamic results in [10]. $Y_{e i}$ is taken to be constant $\left(Y_{e 0}\right)$ for $t_{0}<t_{\mathrm{pb}} \leq t_{1}$ and $Y_{e}\left(t_{\mathrm{pb}}\right)=\left(Y_{e 0}-Y_{e 1}\right)\left(t_{\mathrm{pb}} / t_{1}\right)^{-1}+Y_{e f}$ for $t>t_{1}$, where $t_{1}=4.0 \mathrm{~s}$ and $Y_{e 1}=0.100 . Y_{e 0}$ is taken to be a free parameter, which varies from 0.460 to 0.630 with the interval of 0.005 (35 cases). It should be noted that $Y_{e}$ changes during the $\alpha$-process phase $\left(T_{9} \sim 7-4\right)$, affected by the neutrino capture on free nucleons. The bottom panel of Figure 3 shows 

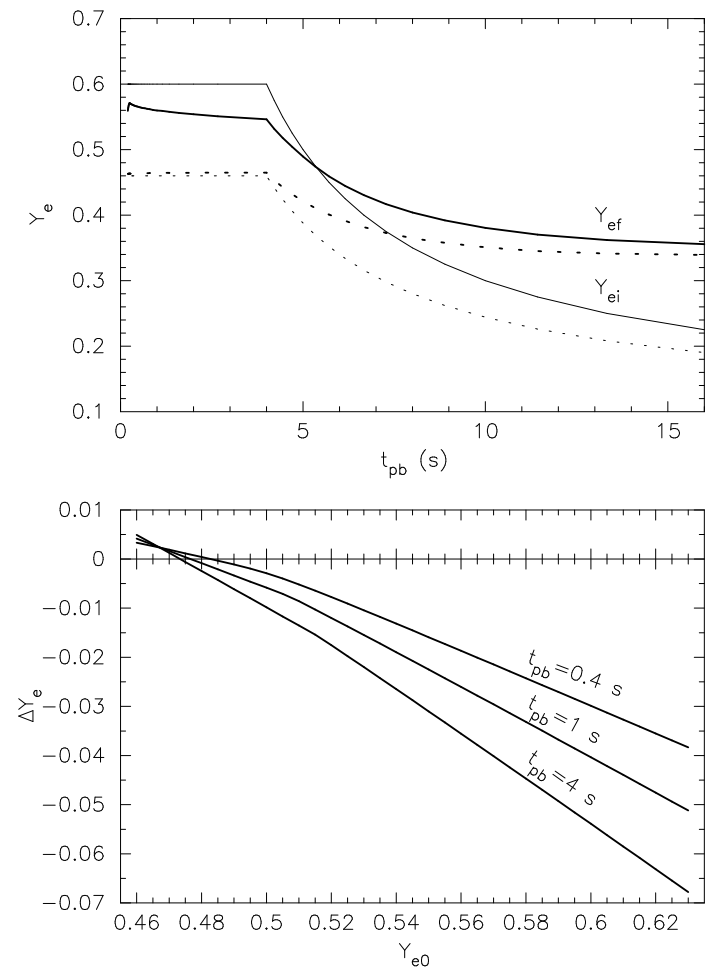

Figure 3: Top: time evolution of $Y_{e i}$ (thin lines) and $Y_{e f} \equiv Y_{e i}+\Delta Y_{e}$ (thick lines) for $Y_{e 0}=0.600$ (solid lines) and 0.460 (dotted lines). Bottom: $\Delta Y_{e}$ at $t_{\mathrm{pb}}=0.4,1.0$, and $4.0 \mathrm{~s}\left(L_{v}=20,8\right.$, and $2 \times$ $10^{51}$ ergs s$^{-1}$, respectively), when the temperature decreases to $T_{9}=3$ (at which the $r p$-process sets in).
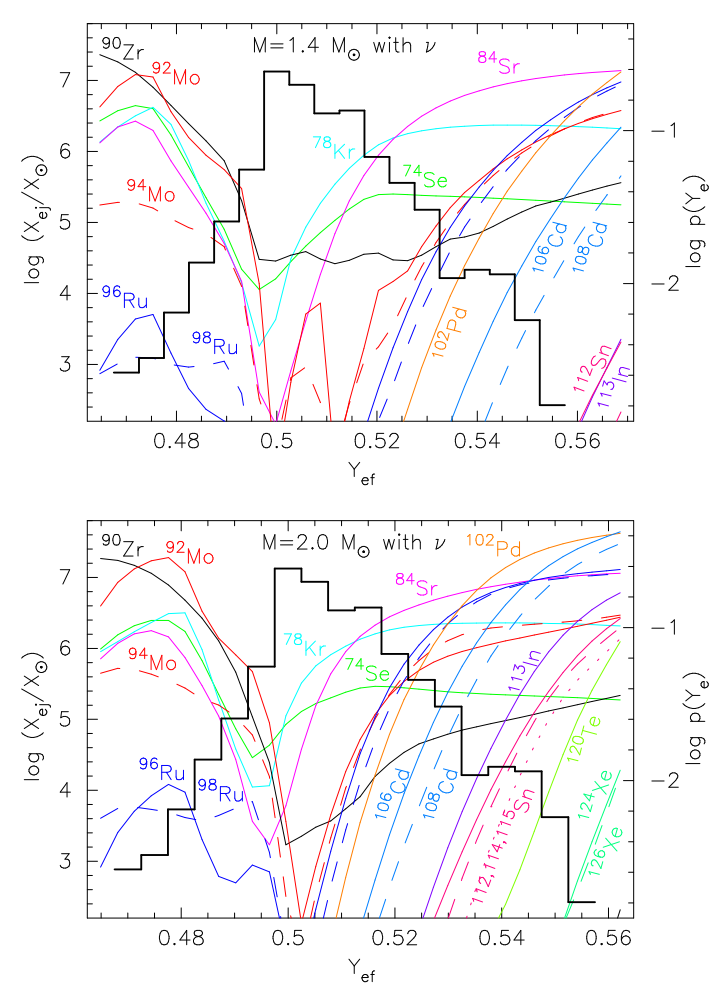

Figure 4: Top: mass-averaged abundances of $p$ nuclei (and ${ }^{90} \mathrm{Zr}$ for comparison purposes) with respect to their solar values for $M=1.4 M_{\odot}$ case as functions of $Y_{e f}\left(t_{\mathrm{pb}}=4.0 \mathrm{~s}\right)$. Also shown is the histogram of the $Y_{e}$ distribution $p\left(Y_{e}\right)$ of the neutrino-processed ejecta in a two-dimensional "exploding" core-collapse simulation by [3]. Bottom: same as the top panel, but for $M=2.0 M_{\odot}$ case.

the shifts $\Delta Y_{e}$ at $t_{\mathrm{pb}}=0.4,1.0$, and $4.0 \mathrm{~s}\left(L_{v}=20,8\right.$, and $2 \times 10^{51} \mathrm{ergs} \mathrm{s}^{-1}$, respectively), when the temperature decreases to $T_{9}=3$ (at which the $r p$-process sets in). The top panel shows $Y_{e i}$ (thin lines) and $Y_{e f} \equiv Y_{e i}+\Delta Y_{e}$ (thick lines) for $Y_{e 0}=0.600$ (solid lines) and 0.460 (dotted lines).

\section{CONTRIBUTION TO THE GALACTIC CHEMICAL EVOLUTION}

In order to examine the contribution of this neutrino-induced $r p$-process to the Galactic chemical evolution, the yields for each $Y_{e i}$ model (35 cases) are mass-averaged over the 54 wind trajectories weighted by $\dot{M}\left(L_{V}\right) \Delta t_{\mathrm{pb}}$. The mass-averaged abundances of $p$-nuclei with respect to their solar values for $M=1.4 M_{\odot}$ (top) and $2.0 M_{\odot}$ (bottom) cases are shown in Figure 4, as functions of $Y_{e f}\left(t_{\mathrm{pb}}=4.0 \mathrm{~s}\right.$ ) (at $L_{v}=2.0 \times 10^{51} \mathrm{ergs} \mathrm{s}^{-1}$, as representative of different $Y_{e f} \mathrm{~s}$ ). Note that the production of $p$-nuclei during later phase $(t>4.0 \mathrm{~s})$ are negligible owing to the neutron richness (Figure 3, top), where the neutron capture nucleosynthesis takes place. As can be seen, a variety of $p$-nuclei are produced with interesting amount for $Y_{e f}>0.5$ models. The heavier $p$-nuclei (up to 

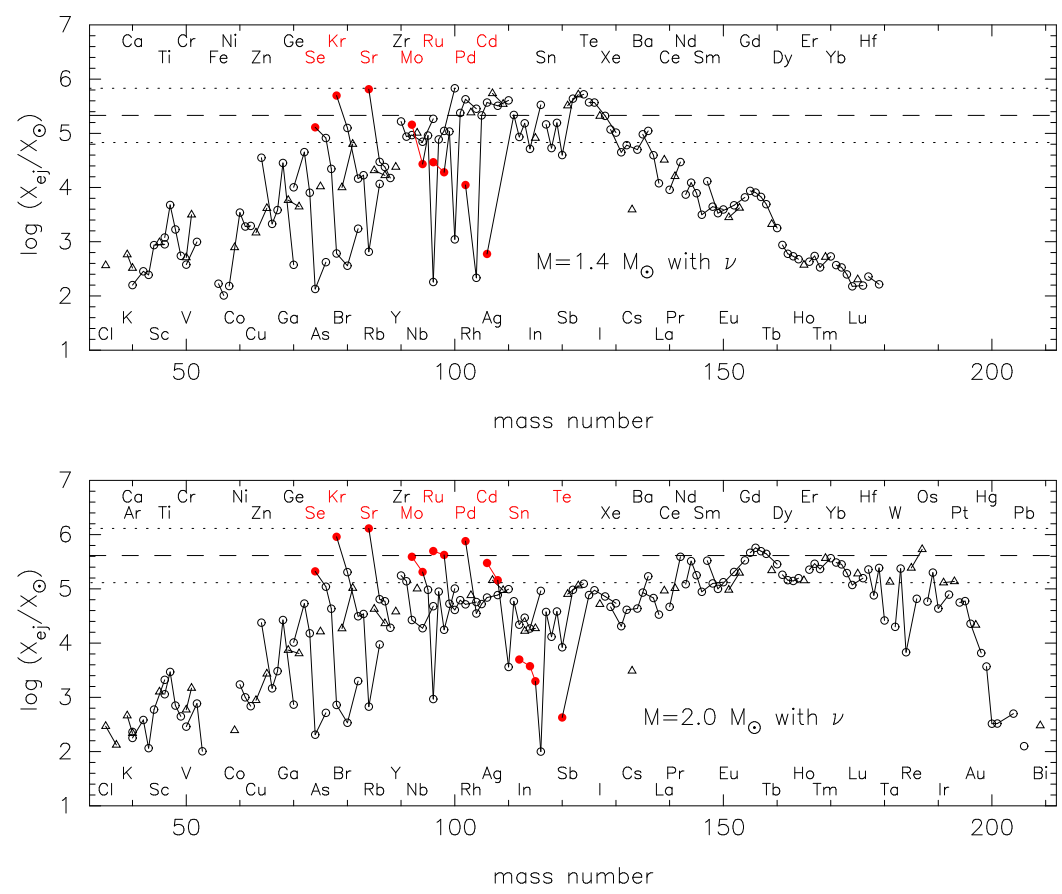

Figure 5: Mass- $Y_{e}$-averaged abundances with respect to their solar values for $M=1.4 M_{\odot}$ (top panel) and $2.0 M_{\odot}$ (bottom panel) cases as functions of mass number. The isotopes (after decay) are denoted by circles (even- $Z$ ) and triangles (odd- $Z$ ). The $p$-nuclei are denoted with filled circles. The solid lines connect isotopes of a given element.

$A \approx 110-120)$ appear for greater $Y_{e f}$ models as well as for larger $M\left(=2.0 M_{\odot}\right)$ case (i.e., greater entropies, see Figure 1). It should be noted that ${ }^{74} \mathrm{Se}$ and ${ }^{92} \mathrm{Mo}$ are most enhanced in slightly neutron-rich compositions (at $Y_{e f} \approx 0.48$, see [6, 12]).

In reality, the neutrino-heated matter must have a certain distribution of $Y_{e}$ as can be seen in a two-dimensional hydrodynamic simulation of core-collapse supernova [3]. The $Y_{e}$ distribution of the neutrino-processed ejecta during the first $468 \mathrm{~ms}$ after core bounce for a $15 M_{\odot}$ progenitor star obtained by [3] is overlaid in Figure 4, which has the maximum at $Y_{e} \approx 0.5$ and dominates in the proton-rich side. To test the contributions of the winds for $M=1.4 M_{\odot}$ and $2.0 M_{\odot}$ cases, the mass-averaged yields for each $Y_{e i}$ model are further $Y_{e}$-averaged $(54 \times 35=1890$ winds in total) with $p\left(Y_{e}\right)$ shown in Figure 4, assuming this distribution to be representative of core-collapse supernovae. The resulting abundances with respect to their solar values are shown in Figure 5 for $M=1.4 M_{\odot}$ (top panel) and $2.0 M_{\odot}$ (bottom panel) cases as functions of mass number. The $p$-nuclei are denoted with filled circles. The dotted horizontal lines indicate a "normalization band" between the largest production factor $\left({ }^{84} \mathrm{Sr}\right)$ and that by a factor of ten less than that, along with a median value (dashed line).

As can be seen in Figure 5, the $p$-nuclei up to ${ }^{92} \mathrm{Mo}$ and ${ }^{108} \mathrm{Cd}$ for $M=1.4 M_{\odot}$ and $2.0 M_{\odot}$ cases, respectively, fall within the normalization band, which are regarded to be the dominant species produced by each event. Note that ${ }^{74} \mathrm{Se}$ and ${ }^{92} \mathrm{Mo}$ are mainly from the slightly neutron-rich ejecta as described above, while other $p$-nuclei are synthesized by the neutrino-induced $r p$-process 
in the proton-rich ejecta. The ejected masses by winds during the first $20 \mathrm{~s}$ are $2.8 \times 10^{-3} M_{\odot}$ and $1.1 \times 10^{-3} M_{\odot}$ for $M=1.4 M_{\odot}$ and $2.0 M_{\odot}$ cases, respectively. Given that the progenitor mass for each case to be, e.g., $15 M_{\odot}$ and $30 M_{\odot}$, respectively, the overproduction factor is expressed as $\sim 10^{-4}\left(X_{\mathrm{ej}} / X_{\odot}\right)$. The requisite overproduction factor of a given isotope for the nucleosynthetic event to be the major source in the solar system is $\sim 10$ [10], assuming that all the core-collapse supernovae produce the same amount of the isotope. The overproduction factors of $\sim 10-100$ (see Figure 5) for the current models imply that the neutrino-driven winds can be potentially the major astrophysical site of these light $p$-nuclei.

The neutron-capture nuclei with $A>100$ result from winds during the later phase $\left(t_{\mathrm{pb}}>4.0 \mathrm{~s}\right.$, see Figure 3, top panel), where the initial compositions are presumed to be neutron rich. In fact, the high-entropy winds of $M=2.0 M_{\odot}$ case are those proposed to be the astrophysical origin of the heavy $r$-process nuclei [7, 13], while that of $M=1.4 M_{\odot}$ to be the origin of light $r$-process nuclei up to $A \sim 130$ [13]. It is interesting to note that no overproduction of the $A \approx 90$ nuclei [7, 10] appears owing to the neutron deficiency in the ejecta [13].

\section{SUMMARY}

It is shown that the interesting amounts of $p$-nuclei can be produced by the neutrino-induced $r p$-process in the proton-rich neutrino-driven winds of core-collapse supernovae. This is due to the presence of free neutrons by the anti-electron neutrino capture on free protons, which bypass the known waiting point nuclei along the nuclear path of the $r p$-process. The nucleosynthesis calculations imply that this neutrino-induced $r p$-process in core-collapse supernovae can be potentially the origin of light $p$-nuclei up to $A \sim 110$, which cannot be easily explained by other astrophysical scenarios.

\section{References}

[1] R. K. Wallace and S. E. Woosley, Astrophys. J. 45, 389 (1981).

[2] O. Koike, M. Hashimoto, K. Arai, and S. Wanajo, Astron. Astrophys., 342, 464 (1999).

[3] R. Buras, M. Rampp, H. -Th Janka, and K. Kifonidis, Astron. Astrophys., 447, 1049 (2006).

[4] J. Pruet, R. D. Hoffman, S. E. Woosley, H. -Th. Janka, and R. Buras, Astrophys. J., 644, 1028 (2006).

[5] C. Fröhlich, et al. Astrophys. J., 637, 415 (2006).

[6] S. Wanajo, Astrophys. J., 647, 1323 (2006).

[7] S. Wanajo, T. Kajino, G. J. Mathews, and K. Otsuki, Astrophys. J., 554, 578 (2001).

[8] S. Wanajo, N. Itoh, Y. Ishimaru, S. Nozawa, and T. C. Beers, Astrophys. J., 577, 853 (2002).

[9] S. Wanajo, Astrophys. J., 650, L79 (2006).

[10] S. E. Woosley, J. R. Wilson, G. J. Mathews, R. D. Hoffman, and B. S. Meyer, Astrophys. J., 433, 229 (1994).

[11] S. Wanajo, S. Goriely, M. Samyn, and N. Itoh, Y., Astrophys. J., 606, 1057 (2004).

[12] R. D. Hoffman, S. E. Woosley, G. M. Fuller, and B. S. Meyer, Astrophys. J., 460, 478 (1996).

[13] S. Wanajo and I. Ishimaru, Nucl. Phys. A, 777, 676 (2006). 